\title{
Phoneutria nigriventer Spider Toxin PnTx2-1 ( $\delta$-Ctenitoxin-Pn1a) Is a Modulator of Sodium Channel Gating
}

\author{
Steve Peigneur ${ }^{1,2, *,+}{ }^{(\mathbb{D}}$, Ana Luiza B. Paiva ${ }^{3,+}$, Marta N. Cordeiro $^{3}$, Márcia H. Borges ${ }^{3}$, \\ Marcelo R. V. Diniz ${ }^{3}$, Maria Elena de Lima ${ }^{2,4}$ and Jan Tytgat ${ }^{1, *}$ \\ 1 Toxicology and Pharmacology, University of Leuven (KU Leuven), Campus Gasthuisberg, P.O. Box 922, \\ Herestraat 49, 3000 Leuven, Belgium \\ 2 Laboratório de Venenos e Toxinas Animais, Dept de Bioquímica e Imunologia, \\ Instituto de Ciências Biológicas, Universidade Federal de Minas Gerais (UFMG), \\ Belo Horizonte 31270-901, Brazil; mariaelena@santacasabh.org.br \\ 3 Departamento de Pesquisa e Desenvolvimento, Fundação Ezequiel Dias, Minas Gerais, \\ Belo Horizonte 30510-010, Brazil; analubpaiva@gmail.com (A.L.B.P.); \\ martadonascimento.phoneutria@gmail.com (M.N.C.); mhborgesb@gmail.com (M.H.B.); \\ mdiniz@funed.mg.gov.br (M.R.V.D.) \\ 4 Programa de Pós-graduação em Ciências da Saúde, Biomedicina e Medicina, \\ Instituto de Ensino e Pesquisa da Santa Casa de Belo Horizonte, Grupo Santa Casa de Belo Horizonte, \\ Minas Gerais, Belo Horizonte 31270-901, Brazil \\ * Correspondence: steve.peigneur@kuleuven.be or stevepeigneur@gmail.com (S.P.); \\ jan.tytgat@kuleuven.be (J.T.); Tel.: +321-632-3404 (J.T.) \\ + These two authors contribute equally to this work.
}

Received: 26 July 2018; Accepted: 16 August 2018; Published: 21 August 2018

Abstract: Spider venoms are complex mixtures of biologically active components with potentially interesting applications for drug discovery or for agricultural purposes. The spider Phoneutria nigriventer is responsible for a number of envenomations with sometimes severe clinical manifestations in humans. A more efficient treatment requires a comprehensive knowledge of the venom composition and of the action mechanism of the constituting components. PnTx2-1 (also called $\delta$-ctenitoxin-Pn1a) is a 53-amino-acid-residue peptide isolated from the venom fraction PhTx2. Although PnTx2-1 is classified as a neurotoxin, its molecular target has remained unknown. This study describes the electrophysiological characterization of PnTx2-1 as a modulator of voltage-gated sodium channels. PnTx2-1 is investigated for its activity on seven mammalian $\mathrm{Na}_{\mathrm{V}}$-channel isoforms, one insect $\mathrm{Na}_{\mathrm{V}}$ channel and one arachnid $\mathrm{Na}_{\mathrm{V}}$ channel. Furthermore, comparison of the activity of both PnTx2-1 and PnTx2-6 on Na 1.5 channels reveals that this family of Phoneutria toxins modulates the cardiac $\mathrm{Na}_{\mathrm{V}}$ channel in a bifunctional manner, resulting in an alteration of the inactivation process and a reduction of the sodium peak current.

Keywords: Phoneutria nigriventer; voltage-gated sodium channel; spider; insecticide; peptide toxin PnTx2-1; gating modifier toxin

Key Contribution: Phoneutria nigriventer toxin PnTx2-1 modulates both the activation and inactivation gating processes of voltage-gated sodium channels. PnTx2-1 exhibits a strong insecticidal activity.

\section{Introduction}

Spiders can be considered one of the most successful venomous animals ever to inhabit the planet with over 47,000 species characterized to date [1]. Despite this diversity, no more than 100 spider species 
have had their venom investigated [2]. With over ten million estimated biologically active peptides in spider venoms [3], this means that less than $0.01 \%$ of spider peptides have been studied $[3,4]$, making spider venom an untapped treasure of biologically active compounds with promising discoveries in drug and bioinsecticide development [1,5,6]. Phoneutria nigriventer are very aggressive, solitary spiders. They are active hunters, relying on their fast-acting and efficient venom for prey capture and defense. They prey normally on insects, although there are reports of Phoneutria hunting on other spiders and small rodents as well [6,7]. Human envenomation by Phoneutria nigriventer is common in Brazil, with 0.5-1\% resulting in severe envenomation, with most of these occurring in children [8]. Cysteine-rich peptide toxins with action on ion channels are the most abundant components in its venom [9]. A recent review describes the complexity of this venom [6]. Historically, the Phoneutria toxins are annotated based on their occurrence in the venom when following the venom purification methods used in the first studies [10], that is, based on a particular chromatographic step and in the order of elution of the toxin, in this step. However, a new nomenclature has been proposed based on the genus of the spider, the target of the toxin and the isoform [11]. According to this nomenclature, PnTx2-1 is also called $\delta$-ctenitoxin-Pn1a and it can be accessed in the Arachnoserver databank (http: / / www.arachnoserver.org) [2]. In-vivo studies have shown that the PhTx2 fraction is toxic to both mice and insects [12]. Nine peptides could be identified from the PhTx2 fraction. These peptides were named accordingly PnTx2-1 to PnTx2-9. PnTx2-1, PnTx2-5 and PnTx2-6 showed higher toxicity after intracerebral (i.c.) injection in mice [13]. Both PnTx2-5 and PnTx2-6 have been characterized as modulators of the inactivation of voltage-gated sodium $\left(\mathrm{Na}_{\mathrm{V}}\right)$ channels $[14,15]$. Toxin PnTx2-1 (5838.8 Da) shares up to 77\% identity with PnTx2-5 and PnTx2-6 (Figure 1) [13]. When injected in mice, PnTx2-1 causes pruritus, lacrimation, increased salivation, sweating and agitation followed by spastic paralysis of the limbs [12]. A recent transcriptomic and proteomic investigation of $P$. nigriventer venom showed that PnTx2-1, altogether with its isoforms, is among the most expressed toxins in this venom [9], suggesting that this toxin may play an important role in the envenoming of natural preys. Based on the in-vivo tests in mice and the sequence homology with PnTx2-5 and PnTx2-6, this peptide is classified as a $\mathrm{Na}_{V}$-channel toxin. However, it has never been tested on any $\mathrm{Na}_{\mathrm{V}}$-channel isoform. Therefore, in this study the $\mathrm{Na}_{V}$-channel subtype selectivity and species specificity of PnTx2-1 are investigated. Furthermore, comparison of the activity of both PnTx2-1 and PnTx2-6 on Nav1.5 reveals that this family of Phoneutria toxins modulates the cardiac $\mathrm{Na}_{\mathrm{V}}$ channel in a bifunctional manner, resulting in an alteration of the inactivation process and a reduction of the sodium peak current.

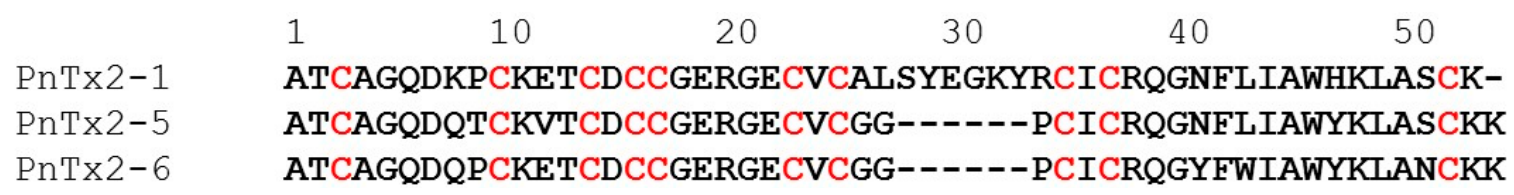

Figure 1. Sequence alignment of PnTx2-1 with $\mathrm{Na}_{\mathrm{V}}$-channel toxins from Phoneutria nigriventer venom fraction 2.

\section{Results}

\subsection{Electrophysiological Charaterization}

\subsubsection{Activity of PnTx2-1 on Mammalian $\mathrm{Na}_{\mathrm{V}}$ Channels}

PnTx2-1 was screened against a panel of seven mammalian $\mathrm{Na}_{\mathrm{V}}$ channel isoforms $\left(\mathrm{Na}_{\mathrm{V}} 1.1-\mathrm{Na}_{\mathrm{V}} 1.6\right.$ and $\left.\mathrm{Na}_{\mathrm{V}} 1.8\right)$, one insect from the cockroach Blattella germanica $\left(\mathrm{BgNa}_{\mathrm{V}} 1\right)$ and one arachnid channel from the mite Varroa destructor $\left(\mathrm{VdNa}_{\mathrm{V}} 1\right)$ (Figure 2). PnTx2-1 was investigated for its activity on these $\mathrm{Na}_{V}$ channels because of their ability to be effectively expressed in oocytes, and these are the most commonly screened $\mathrm{Na}_{V}$ channels when testing for $\mathrm{Na}_{V}$ channel effects of peptide toxins [16]. 
$\mathrm{Na}_{\mathrm{v}} \mathbf{1 . 1}$

A

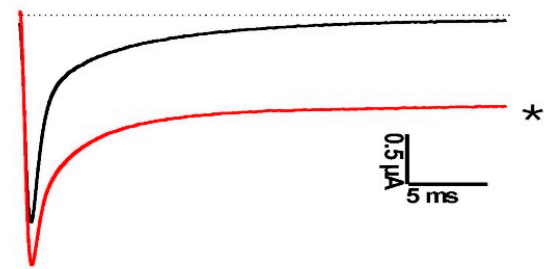

$\mathrm{Na}_{\mathrm{v}} \mathbf{1 . 3}$

C

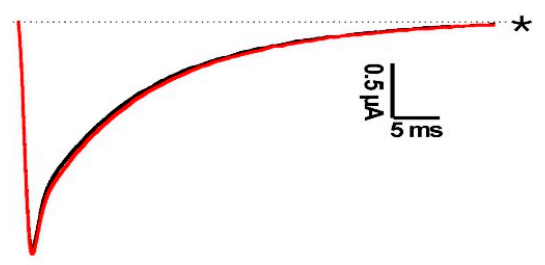

$\mathrm{Na}_{\mathrm{v}} \mathbf{1 . 5}$

E

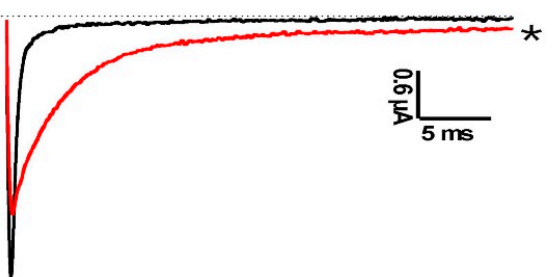

G

$\mathrm{Na}_{\mathrm{v}} \mathbf{1 . 8}$

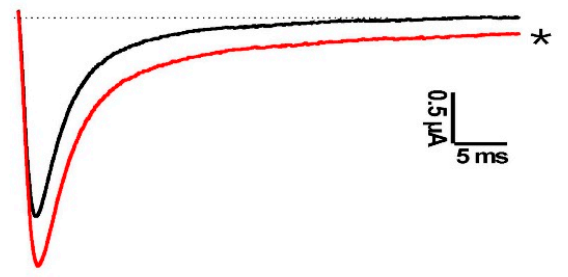

I

$\mathrm{BgNa}_{\mathrm{v}} 1$

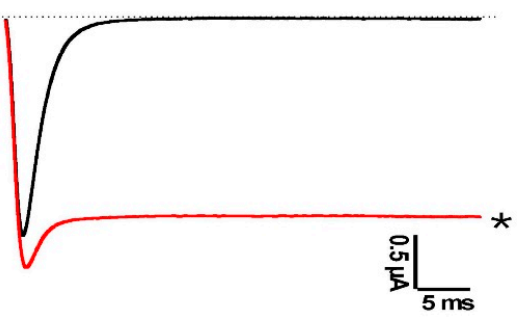

$\mathrm{Na}_{\mathrm{v}} \mathbf{1 . 2}$

B

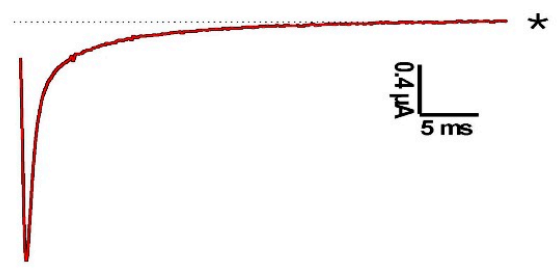

D

$\mathrm{Na}_{\mathrm{v}} \mathbf{1 . 4}$

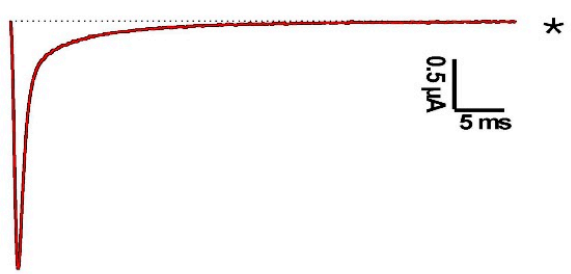

$\mathrm{Na}_{\mathrm{v}} \mathbf{1 . 6}$

$\mathbf{F}$

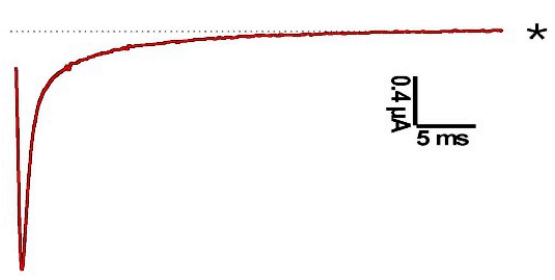

H

$\mathrm{VdNa}_{\mathrm{v}} \mathbf{1}$
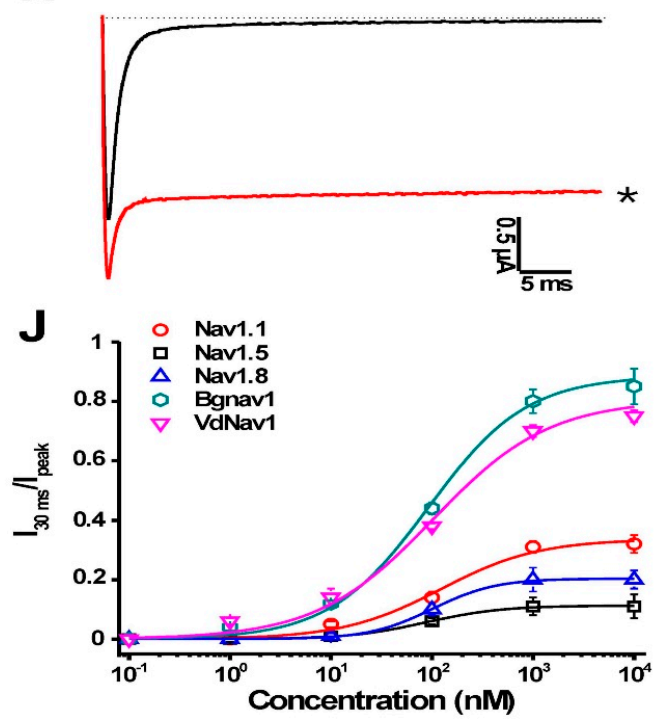

Figure 2. Electrophysiological profiles of PnTx2-1 on Na $\mathrm{N}_{\mathrm{V}}$ s. Panels show superimposed current traces of $1 \mu \mathrm{M}$ PnTx2-1. The dotted line indicates zero current level. Black, current trace in control conditions; red, current trace in toxin situation. The asterisk marks steady-state current trace after application of $1 \mu \mathrm{M}$ peptide. The last panel shows the concentration-response curves for PnTx2-1 on different $\mathrm{Na}_{\mathrm{V}}$ channel isoforms.

Among the mammalian isoforms, $1 \mu \mathrm{M}$ PnTx2-1 slowed the inactivation of $\mathrm{Na}_{\mathrm{V}} 1.1, \mathrm{Na}_{\mathrm{V}} 1.5$ and $\mathrm{Na}_{V}$ 1.8. $\mathrm{Na}_{V} 1.2, \mathrm{Na}_{V} 1.3, \mathrm{Na}_{V} 1.4$ and $\mathrm{Na}_{V} 1.6$ were not affected by PnTx2-1. Interestingly, PnTx2-1 had a profound effect on the inactivation of the insect channel $\mathrm{BgNa}_{\mathrm{V}} 1$ and the arachnid channel $\mathrm{VdNa} \mathrm{V}_{\mathrm{V}} 1$. 
PnTx2-1 completely inhibited inactivation of $\mathrm{BgNa}_{\mathrm{V}} 1$ channels, resulting in sustained non-inactivating currents. Concentration-response curves were constructed to determine the values at which half of the channels were modulated by PnTx2-1. Among the mammalian isoforms, the half-maximal effective concentration $\left(\mathrm{EC}_{50}\right)$ values yielded $122.1 \pm 34.6 \mathrm{nM}, 87.0 \pm 7.5 \mathrm{nM}$ and $101.1 \pm 5.1 \mathrm{nM}$ for $\mathrm{Na}_{\mathrm{V}} 1.1$, $\mathrm{Na}_{V} 1.5$ and $\mathrm{Na}_{V} 1.8$, respectively. Activation and steady-state inactivation curves were constructed for $\mathrm{Na}_{V} 1.1, \mathrm{Na}_{V} 1.5$ and $\mathrm{Na}_{V} 1.8$ channels (Figure $3 \mathrm{~A}-\mathrm{C}$, Table 1 ). In the presence of $1 \mu \mathrm{M}$ toxin, a significant modulation of gating kinetics was observed for $\mathrm{Na}_{V} 1.1$ and $\mathrm{Na}_{V} 1.5$ (Figure 3A,B, Table 1). For both $\mathrm{Na}_{V} 1.1$ and $\mathrm{Na}_{V} 1.5$ channels, the midpoint of activation shifted towards more depolarized potentials. At the same time, the steady-state inactivation curves shifted towards more hyperpolarized membrane potentials. This resulted in an increased window of open probability for both $\mathrm{Na}_{V} 1.1$ and $\mathrm{Na}_{V} 1.5$. No relevant modulation of the gating kinetics was observed for $\mathrm{Na}_{\mathrm{V}} 1$.8-channel isoforms since no significant shift in the half-maximal activation voltage $\left(\mathrm{V}_{1 / 2}\right)$ values was noted (Figure $3 \mathrm{C}$, Table 1 ). No significant change in the reversal potential was observed in the presence of toxin which indicates that the ion selectivity of $\mathrm{Na}_{V}$ channels is not altered. One micromolar PnTx2-1 strongly enhanced the recovery from inactivation for $\mathrm{Na}_{V} 1.5$ channels with $\tau=50.2 \pm 3.0 \mathrm{~ms}$ in control conditions and $\tau=22.8 \pm 3.2 \mathrm{~ms}$ in the presence of the toxin (Figure 3D). To verify whether PnTx2-1 binds to neurotoxin site 1, competitive binding experiments were performed. Application of tetrodotoxin (TTX) at its half-maximal inhibitory concentration $\left(\mathrm{IC}_{50}\right)$ resulted in a blockage of half of the expressed $\mathrm{Na}_{\mathrm{V}} 1.5$ channels. This was observed as a 50\% decrease of the sodium current peak amplitude. An additional reduction of the peak amplitude was the result of subsequent and additional application of PnTx2-1 at its $\mathrm{EC}_{50}(n=6)$ (Figure 3E).

Table 1. $\mathrm{V}_{1 / 2}$ values for the activation and steady-state inactivation curves obtained for $\mathrm{Na}_{\mathrm{V}} 1.1, \mathrm{Na}_{\mathrm{V}} 1.5$, $\mathrm{Na}_{V} 1.8$ and $\mathrm{BgNa}_{V} 1$.

\begin{tabular}{ccccc}
\hline & \multicolumn{2}{c}{ Activation } & \multicolumn{2}{c}{ Inactivation } \\
\hline $\mathrm{V}_{1 / 2}(\mathrm{mV})$ & Control & $\begin{array}{c}\text { PnTx2-1 } \\
(1 \mu \mathrm{M})\end{array}$ & Control & $\begin{array}{c}\text { PnTx2-1 } \\
(1 \mu \mathrm{M})\end{array}$ \\
$\mathrm{Na} \mathrm{a}_{\mathrm{V}} 1.1$ & $-56.4 \pm 0.2$ & $-63.4 \pm 0.1$ & $-54.7 \pm 0.1$ & $-45.3 \pm 0.1$ \\
$\mathrm{Na} 1.5$ & $-53.1 \pm 0.1$ & $-45.3 \pm 0.1$ & $-78.4 \pm 0.2$ & $-87.5 \pm 0.7$ \\
$\mathrm{Na}_{\mathrm{V}} 1.8$ & $-11.5 \pm 0.1$ & $-11.4 \pm 0.1$ & $-55.8 \pm 0.4$ & $-63.9 \pm 0.4$ \\
$\mathrm{BgNa}_{\mathrm{V}} 1$ & $-38.1 \pm 0.1$ & $-45.8 \pm 0.3$ & $-63.3 \pm 0.3$ & $-70.7 \pm 0.13$ \\
\hline
\end{tabular}

\subsubsection{Activity of PnTx2-1 on Insect $\mathrm{Na}_{\mathrm{V}}$-Channel Currents}

The $\mathrm{EC}_{50}$ values for $\mathrm{BgNa}_{\mathrm{V}} 1$ and $\mathrm{VdNa}_{\mathrm{V}} 1$ were determined at $91.9 \pm 17.0 \mathrm{nM}$ and $101.8 \pm 14.7 \mathrm{nM}$, respectively. The insect $\mathrm{Na}_{V}$ channel $\mathrm{BgNa}_{V} 1$ was used to further investigate the characteristics of PnTx2-1 in inducing modulation of channel gating (Figure 4). A shift in the midpoint of activation and in the voltage dependence of steady-state inactivation was observed (Table 1). From Figure 4A, it can be seen that the steady-state inactivation became incomplete after toxin addition. A $62.3 \pm 3.2 \%$ non-inactivating current component of steady-state inactivation can be seen. In addition, we examined the recovery from fast inactivation in the absence or presence of PnTx2-1 and found that $1 \mu \mathrm{M}$ does not influence the recovery from fast inactivation in $\mathrm{BgNa}_{\mathrm{V}} 1$ (Figure $4 \mathrm{~B}$ ). Considering the incomplete steady-state inactivation of $\mathrm{BgNa}_{\mathrm{V}} 1$ channels in the presence of PnTx2-1, we decided to determine whether inhibition occurs during the closed or open state of the channel. One micromolar toxin was applied to the bath solution with oocytes clamped at $-90 \mathrm{mV}$, allowing interaction with the membrane for $2 \mathrm{~min}$ without depolarizing the membrane. After $2 \mathrm{~min}$, currents were elicited by a depolarizing pulse to $0 \mathrm{mV}$. The obtained currents in the presence of toxin were normalized to the currents obtained in the same cells in control conditions. A significant slowing down of inactivation was observed when PnTx2-1 was applied to the oocytes without applying depolarizing pulses (Figure 4C). This indicates that there is toxin interaction with the channel in the closed state and suggests that membrane depolarization and hence most likely channel opening is not required to allow the toxin to bind. 

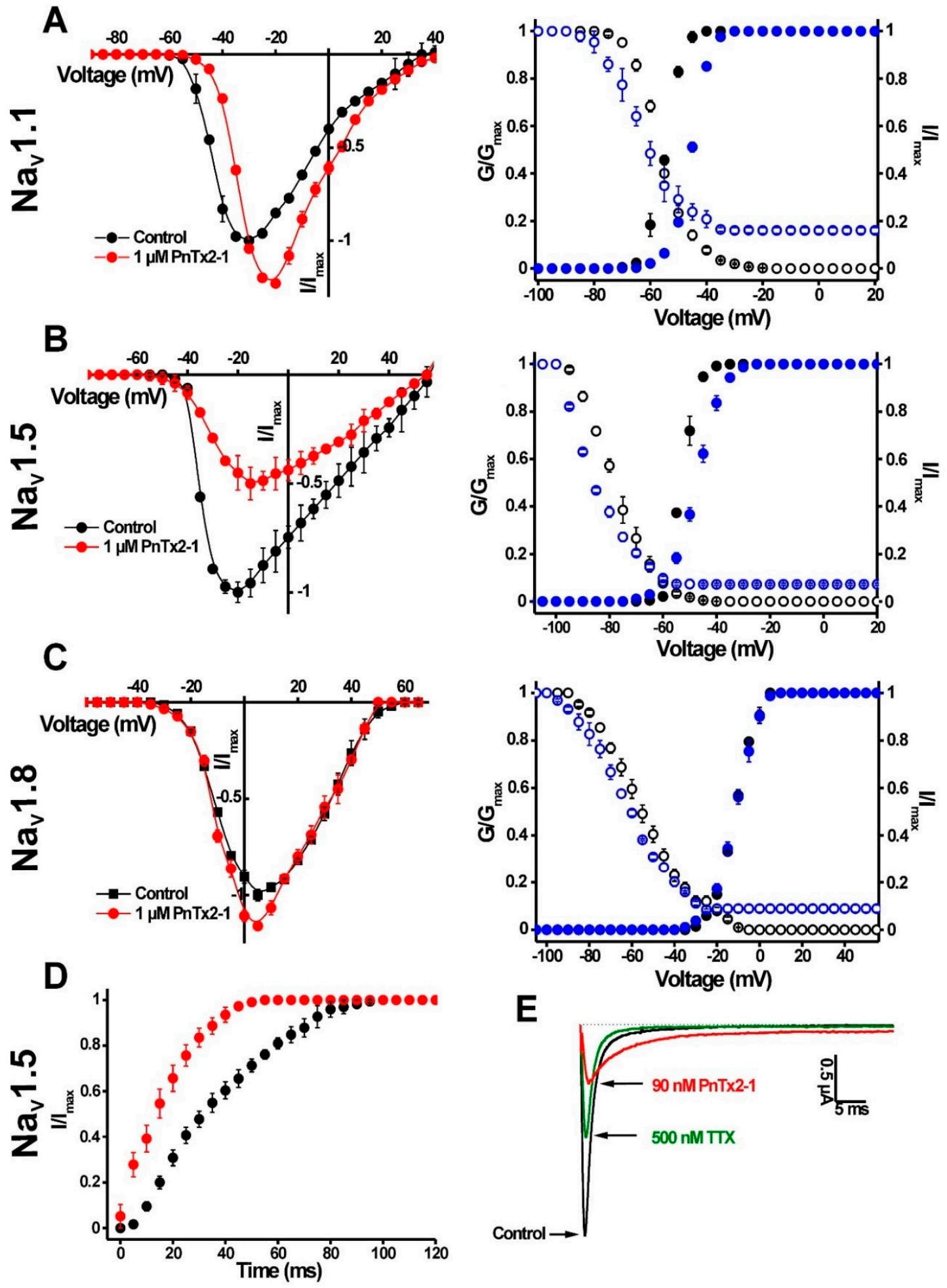

Figure 3. Electrophysiological characterization of PnTx2-1 on mammalian $\mathrm{Na}_{\mathrm{V}}$ channels. Left panels show the current voltage relationships and the right panels show the steady-state activation (closed symbols) and inactivation (open symbols) curves in control (black) and toxin conditions (1 $\mu \mathrm{M}$ PnTx2-1, blue) for $\mathrm{Na}_{V} 1.1$ (A), $\mathrm{Na}_{V} 1.5$ (B) and $\mathrm{Na}_{V} 1.8$ (C). (D) Recovery from inactivation for $\mathrm{Na}_{V} 1.5$ channels. Control conditions (black symbols) and in the presence of $1 \mu \mathrm{M}$ PnTx2-1 (red symbols) are shown. (E) Competitive experiments to indicate that PnTx2-1 does not bind at site 1. Representative traces for $\mathrm{Na}_{\mathrm{V}} 1.5$ channels are shown in control; after application of $500 \mathrm{nM}$ tetrodotoxin (TTX) and after subsequent addition of 90 nM PnTx2-1. 

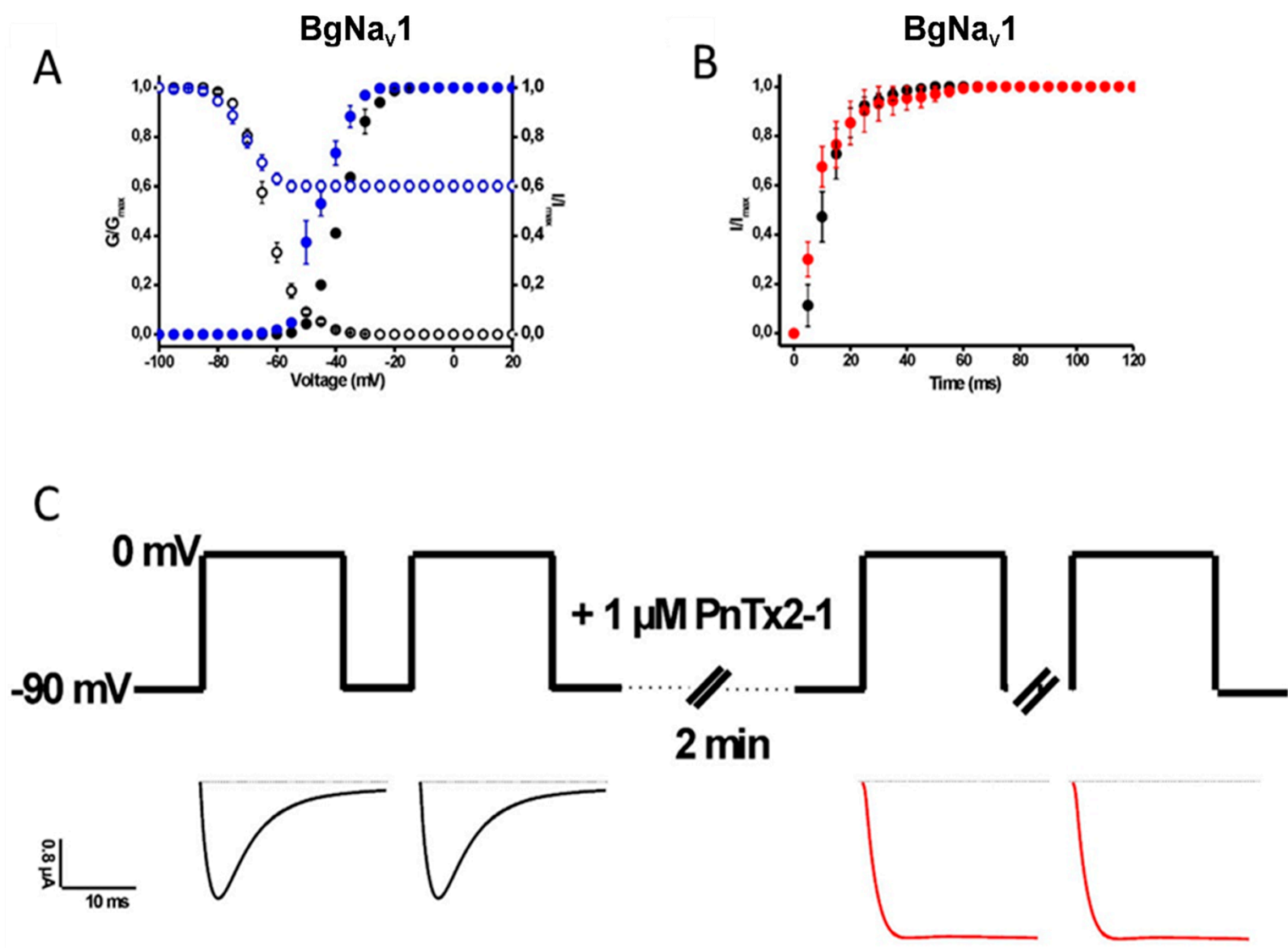

Figure 4. Electrophysiological characterization of PnTx2-1 on insect $\mathrm{Na}_{V}$ channels. (A) Steady-state activation (closed symbols) and inactivation (open symbols) curves in control (black) and toxin conditions (1 $\mu \mathrm{M}$ PnTx2-1, blue) for $\mathrm{BgNa}_{\mathrm{V}}$ 1.1. (B) Recovery from inactivation in control (black symbols) and in the presence of $1 \mu \mathrm{M}$ PnTx2-1 (red symbols). (C) Investigation of the state-dependence of indicating that an expected degree of channel inactivation inhibition was observed after the $2 \mathrm{~min}$ incubation, indicating that the open state is not required for toxin interaction with the channel.

\subsubsection{Activity of PnTx2-6 on Nav1.5-Channel Currents}

The pharmacological phenotype induced on $\mathrm{Na}_{\mathrm{V}} 1.5$ channels by PnTx2-1 is very similar to what has been reported previously for PnTx2-6 [17]. Therefore, we performed further characterisation of PnTx2-6 on $\mathrm{Na}_{\mathrm{V}} 1.5$ channels by investigating the current-voltage relationship in the presence of PnTx2-6. In the presence of $1 \mu \mathrm{M}$ PnTx2-6, an inhibition of the sodium peak current and a delay of the inactivation was observed (Figure 5A). The slowing down of inactivation was characterized by an $\mathrm{EC}_{50}$ value of $22.3 \pm 3.1 \mathrm{nM}$ (Figure $5 \mathrm{~B}$ ). The voltage-current relationship shows a reduction of the sodium current by $21.3 \pm 2.8 \%$ in the presence of $200 \mathrm{nM}$ PnTx2-6 (Figure 5C). Construction of the activation and steady-state inactivation curves indicated that no significant modification of gating processes occurs (Figure 5D). 
A
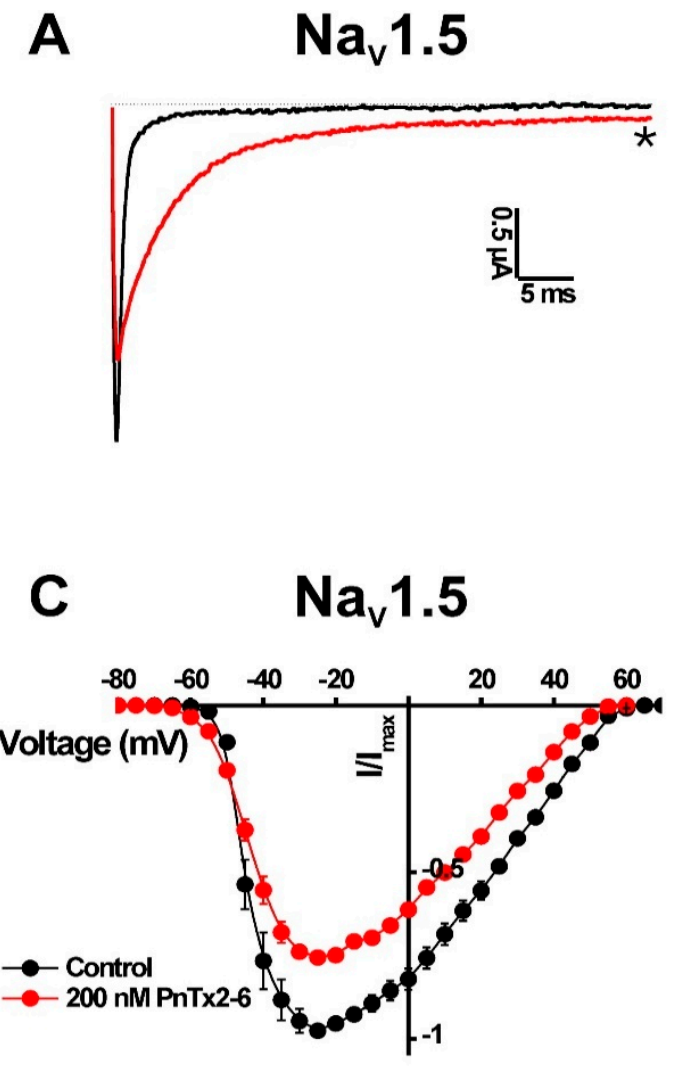

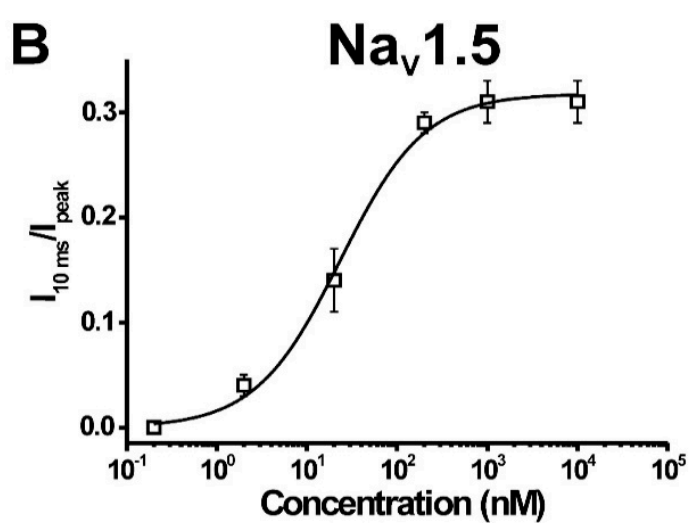

D $\quad \mathrm{Na}_{\mathrm{v}} 1.5$

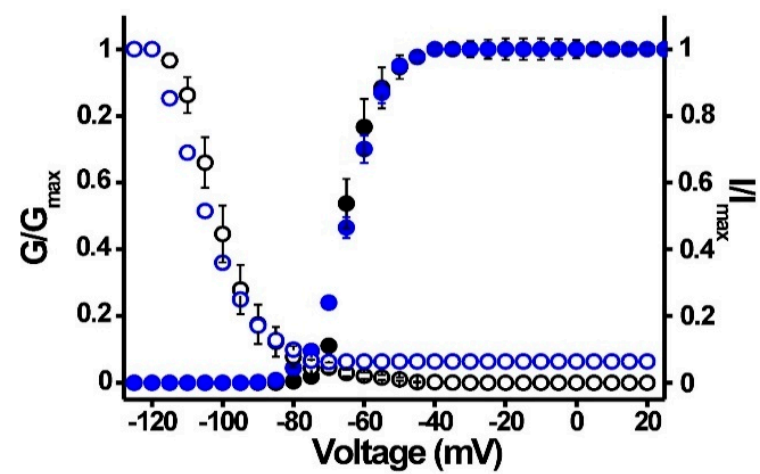

Figure 5. Electrophysiological characterization of PnTx2-6 on $\mathrm{Na}_{\mathrm{V}} 1.5$. (A) Representative whole-cell current traces in control (black) and toxin (red) conditions are shown. The dotted line indicates the zero-current level. The asterisk marks steady-state current trace after application of $1 \mu \mathrm{M}$ peptide. (B) Concentration-response curve for PnTx2-6 on Nav1.5. (C) Current-voltage relationship in control conditions and in the presence of $200 \mathrm{nM}$ PnTx2-6. (D) Steady-state activation (closed symbols) and inactivation (open symbols) curves in control (black) and toxin conditions (1 $\mu$ M PnTx2-6, blue) for $\mathrm{Na}_{\mathrm{V}} 1.5$ channels.

\section{Discussion}

PnTx2-1 shares the highest sequence identity with PnTx2-5 and PnTx2-6, which are also isolated from the Phoneutria nigriventer venom fraction 2 [13]. PnTx2-5 and PnTx2-6 are the best-characterized toxins from the PhTx2 venom fraction $[14,15,17,18]$. These two toxins share $90 \%$ sequence identity, with only five amino acid residues different (Figure 1). PnTx2-5 and PnTx2-6 are responsible for the painful and persistent penile erection, also known as priapism, a common clinical manifestation observed upon Phoneutria nigriventer envenomation [18,19]. Both peptides modulate sodium channel kinetics by slowing down the inactivation process and by shifting the voltage dependence of activation towards more hyperpolarized potentials [14,15]. Interestingly, significant differences in potency have been reported despite the high homology between both peptides. PnTx2-5 displayed an approximately six-fold lower potency than PnTx2-6 on macroscopic sodium currents in patch clamp experiments using GH3 cells [20]. It has been suggested that Tyr41 and Trp43 in PnTx2-6 are involved in the higher potency compared to PnTx2-5 [14]. Interestingly, these residues are also not conserved in PnTx2-1 (Figure 1). Nevertheless, similar EC 50 values were found for PnTx2-1 and PnTx2-6 on Nav1.5 channels (Figures 2J and 5B). While PnTx2-6 exerts a strong affinity for $\mathrm{Na}_{\vee} 1.2-\mathrm{Na}_{\mathrm{V}} 1.4$ and $\mathrm{Na}_{\mathrm{V}} 1.6$ channels [17], PnTx2-1 shows no activity on these channels, even at concentrations up to $5 \mu \mathrm{M}$ (data not shown). It is well established that even minor differences in amino acid composition of peptide toxins can 
influence the $\mathrm{Na}_{\mathrm{V}}$ channel isoform selectivity [21,22]. Binding experiments in brain synaptosomes indicated a partial competition between the $\alpha$-scorpion toxin AaHII (from Androctonus australis Hector) and PnTx2-6 [14]. It thus can be hypothesized that PnTx2-1 also acts by binding to a site similar to the site that PnTx2-6 and the typical $\alpha$-scorpion toxins bind to. $\beta$-Scorpion toxins and certain spider toxins modulate $\mathrm{Na}_{\mathrm{V}}$ channels by shifting the midpoint of activation towards more negative potentials, often resulting in a reduction in sodium peak current [23-25]. However, the $\beta$-scorpion toxin CssIV (from Centruroides suffusus suffusus) does not compete with PnTx2-6. Therefore, it seems unlikely that PnTx2-6 shares a binding site with $\beta$-scorpion toxins [14]. However, it can be suggested that the PnTx2-1- and PnTx2-6-induced inhibition of the sodium peak current (Figures 2E, 3B and 5A) results from gating modification, rather than physical obstruction of the ion pathway. Scorpion toxins such as Ts1 (from Tityus serrulatus) and Tz1 (from Tityus zulianus) induce a similar pharmacological phenotype activity on $\mathrm{Na}_{\mathrm{V}} 1.5$ channels to that observed for PnTx2-1 [23,25,26]. For these toxins, it was evidenced that by trapping the voltage sensor in the inward position, channels are prevented from opening, which is seen as an inhibition of the sodium current for Nav1.5 channels $[16,23,25,26]$.

$\delta$-Atracotoxins $(\delta-\mathrm{ACTX})$ are a family of $\mathrm{Na}_{\mathrm{V}}$ channel-targeting toxins isolated from the venom of Australian funnel-web spiders [24]. They are classified in the $\mathrm{Na}_{V}$ channel-targeting spider toxin (NaSpTx) family 4. This family constitutes six peptides isolated from Australian funnel-web spiders and one peptide from the Asian spider Macrothele gigas. They are peptides of 42 amino acid residues including eight conserved cysteine residues which form four disulfide bridges in an inhibitor cysteine-knot (ICK) motif [24,27]. Although very low sequence homology $(<25 \%)$ can be found between PnTx2-1 and these funnel-web spider toxins, they do seem to modulate the $\mathrm{Na}_{\mathrm{V}}$ channels in a similar way. In patch-clamp experiments, it was shown that $\delta$-ACTX interact with TTX-sensitive but not with TTX-resistant sodium currents in dorsal root ganglion (DRG) neurons. To date, no information is available on the $\mathrm{Na}_{\mathrm{V}}$-channel subtype selectivity of $\delta$-ACTX since these peptides have not yet been tested on heterologously expressed $\mathrm{Na}_{\mathrm{V}}$-channel isoforms. However, in DRGs, $\delta$-ACTX produce a selective slowing of $\mathrm{Na}_{\mathrm{V}}$-current inactivation and a reduction in peak sodium current. Moreover, they cause a hyperpolarizing shift in the midpoint of activation, and an increased recovery from inactivation was seen for toxin-bound channels [20,28-30]. Radiolabeled binding assays have determined that the $\delta$-ACTX bind to the neurotoxin site 3 [31]. Several residues have been suggested to be important for the interaction of $\delta$-ACTX with the $\mathrm{Na}_{\mathrm{V}}$ channels. However, this family still awaits structure-activity data to elucidate the exact key residues [4,24]. It thus seems that PnTx2-1 and the $\delta$-ACTX exert a comparable complex voltage-dependent modulation of $\mathrm{Na}_{\mathrm{V}}$ channel gating.

Although both toxins were insecticidal to the larval and adult forms of the housefly [13], PnTx2-5 and PnTx2-6 have never been evaluated for their insecticidal activity towards insect $\mathrm{Na}_{\mathrm{V}}$ channels. In fact, it was always believed that Phoneutria nigriventer venom fraction 4 (PhTx4) was the venom fraction with the highest and most specific insecticidal activity [6,32]. However, here we show that peptides of $\mathrm{PhTx} 2$ fraction have a similar insecticidal potential, at least regarding $\mathrm{Na}_{\mathrm{V}}$ channels. PnTx2-1 is the first member of this spider toxin family to be characterized in depth for its activity on insect $\mathrm{Na}_{\mathrm{V}}$ channels. The weak species selectivity of PnTx2-1 challenges the suitability of this peptide for the development of novel insecticidal agents.

\section{Conclusions}

The characterization of PnTx2-1 as a $\mathrm{Na}_{\mathrm{V}}$-channel modulator adds to the expanding knowledge on the Phoneutria nigriventer venom. A better understanding of the mechanism of action of its composing venom peptides is important for an effective treatment of Phoneutria nigriventer envenomation. This work reports for the first time the activity of a toxin isolated from the Phoneutria nigriventer venom fraction 2 (PhTx2) on insect $\mathrm{Na}_{\mathrm{V}}$ channels. This observation indicates that besides venom fraction 4 , fraction 2 might also be considered as a source of insecticidal peptides. 


\section{Materials and Methods}

\subsection{Toxin Purification}

Crude venom from $P$. nigriventer was collected from mature male and female spiders maintained in the Scientific Arachnidium at the Fundacion Ezequiel Dias (FUNED) in Belo Horizonte, Brazil (Sisgen: License for Access to Genetic Patrimony Number 010815/2015-5). Toxin PnTx2-1 was purified as previously described [13].

\subsection{Electrophysiology}

\subsubsection{Heterologous Expression in Xenopus laevis oocytes}

For expression in $X$. laevis oocytes, the plasmids encoding the $\alpha$-subunits $\mathrm{Na}_{\mathrm{V}} 1.1-\mathrm{Na} \mathrm{V}_{\mathrm{V}} 1.6, \mathrm{Na}_{\mathrm{V}} 1.8$, cockroach Blattella germanica BgNa 1.1 , Varroa destructor $\mathrm{VdNa}_{\mathrm{V}} 1$, and the corresponding $\beta$-subunits, $\mathrm{r} \beta 1, \mathrm{~h} \beta 1$ and Drosophila melanogaster TipE, were linearized with the respective restriction enzymes, mentioned between parentheses, and transcribed using the T7 or SP6 mMESSAGE-mMACHINE transcription kit (Ambion, Austin, TX, USA). Stage V and VI oocytes were harvested from anesthetized female X. laevis frogs, as described previously [33]. Oocytes were injected with 30-50 nL of 1-3 $\mu \mathrm{g} / \mu \mathrm{L}$ $\mathrm{Na}_{V}$-channel cRNA using a microinjector (Drummond Scientific, Broomall, PA, USA). The oocytes were then incubated in ND96 solution (in mM: $\mathrm{NaCl} 96, \mathrm{KCl} 2, \mathrm{MgCl}_{2}$ 1, $\mathrm{CaCl}_{2}$ 1.8, HEPES 5), adjusted to $\mathrm{pH} 7.5$ and supplemented with $50 \mathrm{mg} / \mathrm{L}$ of gentamycin sulphate and $90 \mathrm{mg} / \mathrm{L}$ theophylline, at $16{ }^{\circ} \mathrm{C}$ for $1-5$ days, until expression of ion channels.

\subsubsection{Electrophysiological Recordings}

Electrophysiological recordings were performed as described previously [33]. In brief, whole-cell currents from oocytes were recorded at room temperature $\left(18-22{ }^{\circ} \mathrm{C}\right)$ by the two-electrode voltage clamp technique using a GeneClamp 500 amplifier (Molecular Devices, Sunnyvale, CA, USA) controlled by a pClamp data acquisition system (Molecular Devices). Oocytes were placed in a bath containing ND96 solution. Voltage and current electrodes were filled with $3 \mathrm{M} \mathrm{KCl}$, and the resistances of both electrodes were kept between 0.7 and $1.5 \mathrm{M} \Omega$. The elicited currents were sampled at $20 \mathrm{kHz}$ and filtered at $2 \mathrm{kHz}$ using a four-pole, low-pass Bessel filter. To eliminate the effect of the voltage drop across the bath grounding electrode, the bath potential was actively controlled by a two-electrode bath clamp. Leak subtraction was performed using a $-\mathrm{P} / 4$ protocol. Whole-cell current traces were evoked every $5 \mathrm{~s}$ by a 100-ms depolarization to the voltage corresponding to the maximal activation of the $\mathrm{Na}_{\mathrm{V}}$ subtype in control conditions, starting from a holding potential of $-90 \mathrm{mV}$. The oocyte expressing the specific $\mathrm{Na}_{\mathrm{V}}$-channel isoform with no toxin added to the bath was considered as control. Concentration-response curves were constructed by adding different toxin concentrations directly to the bath solution. The percentage of $\mathrm{Na}_{\mathrm{V}}$ modulation was plotted against the logarithm of the applied concentrations and fitted with the Hill equation:

$$
\mathrm{y}=100 /\left[1+\left(\mathrm{IC}_{50} /[\text { toxin }]\right)\right] \times \mathrm{h}
$$

where $\mathrm{y}$ is the amplitude of the toxin-induced effect, $\mathrm{IC}_{50}$ is the toxin concentration at half-maximal efficacy, [toxin] is the toxin concentration and $h$ is the Hill coefficient. The amplitude of the toxin-induced effect is obtained by dividing the current amplitude at $30 \mathrm{~ms}$ in steady-state toxin situation by the peak current amplitude in control conditions. To investigate the effects on the voltage dependence of activation, current traces were induced by $100-\mathrm{ms}$ depolarizations from a holding potential of -90 to $65 \mathrm{mV}$ with 5-mV increments. To investigate the effects on the steady-state inactivation process, oocytes were depolarized using a standard two-step protocol. From a holding potential of $-90 \mathrm{mV}, 100-\mathrm{ms}$ prepulses were generated, ranging from -90 to $65 \mathrm{mV}$ with 5-mV 
increments, immediately followed by a $100-\mathrm{ms}$ test pulse to $0 \mathrm{mV}$. Data were normalized to the maximal $\mathrm{Na}^{+}$current amplitude, plotted against prepulse potential and fitted using the Boltzmann equation:

$$
\mathrm{I}_{\mathrm{Na}} / \mathrm{I}_{\max }=[(1-\mathrm{C}) /(1+\exp ((\mathrm{V}-\mathrm{Vh}) / \mathrm{k}))]+\mathrm{C}
$$

where $\mathrm{I}_{\max }$ is the maximal $\mathrm{I}_{\mathrm{Na}}, \mathrm{Vh}$ is the voltage corresponding to half-maximal inactivation, $\mathrm{V}$ is the test voltage, $\mathrm{k}$ is the slope factor, and $\mathrm{C}$ is a constant representing a non-inactivating persistent fraction (close to zero in control). All data were tested for normality using a D'Agustino Pearson omnibus normality test. Data following a Gaussian distribution were analyzed for significance using one-way ANOVA and the Bonferroni test. Nonparametric data were analyzed for significance using the Kruskal-Wallis and Dunn tests. All data were analyzed using pClamp Clampfit 10.4 (Molecular Devices ${ }^{\circledR}$, Downingtown, PA, USA, 2003) and Origin 7.5 software (Originlab ${ }^{\circledR}$, Northampton, MA, USA, 2003) and are presented as mean \pm standard error (SEM) of at least 3 independent experiments $(n \geq 3)$.

Author Contributions: Conceptualization, S.P., A.L.B.P., M.R.V.D., M.E.d.L. and J.T.; Methodology, S.P., A.L.B.P., M.N.C., M.H.B.; Software, S.P.; Formal Analysis, S.P. \& A.L.B.P.; Investigation, S.P. \& A.L.B.P.; Resources, M.R.V.D., M.E.d.L. and J.T.; Data Curation, S.P. \& A.L.B.P.; Writing-Original Draft Preparation, S.P. \& A.L.B.P. Writing-Review \& Editing, S.P., A.L.B.P., M.R.V.D., M.E.d.L. and J.T.; Visualization, S.P. \& A.L.B.P.; Supervision, M.R.V.D., M.N.C., M.H.B., M.E.d.L. and J.T.; Project Administration, M.R.V.D., M.N.C., M.H.B., M.E.d.L. and J.T.; Funding Acquisition, M.R.V.D., M.N.C., M.H.B., M.E.d.L. and J.T.

Funding: This work was supported by the following grants: JT was supported by grant CELSA/17/047-BOF/ISP. $\mathrm{SP}$ is a PhD fellow supported by CAPES (Coordenação de Aperfeiçoamento de Pessoal de Nível Superior). MEdL was supported by grants from CAPES (Coordenação de Aperfeiçoamento de Pessoal de Nivel Superior), CNPq (Conselho Nacional de Desenvolvimento Científico e Tecnológico) and FAPEMIG (Fundação de Pesquisa do Estado de Minas Gerais).

Acknowledgments: The authors thank John N. Wood (University College London, London, UK) for sharing rNaV1.8; A. L. Goldin (University of California, Irvine, CA, USA) for sharing rNaV1.1, rNaV1.2, rNaV1.3, and mNaV1.6; G. Mandel (State University of New York, Stony Brook, NY, USA) for sharing rNaV1.4; R. G. Kallen (Roche Institute of Molecular Biology, Nutley, NJ, USA) for sharing hNaV1.5; K. Dong (Michigan State University, USA) for sharing the BgNaV1.1 and VdNaV1 clones; S. C. Cannon (University of Texas Southwestern Medical Center, Dallas, TX, USA) for sharing the h $\beta 1$ subunit and Martin S. Williamson (Rothhamsted Research, Harpenden, UK) for providing the Para and tipE clone.

Conflicts of Interest: The authors declare no conflict of interest.

\section{References}

1. Pineda, S.S.; Undheim, E.A.; Rupasinghe, D.B.; Ikonomopoulou, M.P.; King, G.F. Spider venomics: Implications for drug discovery. Future Med. Chem. 2014, 6, 1699-1714. [CrossRef] [PubMed]

2. Pineda, S.S.; Chaumeil, P.A.; Kunert, A.; Kaas, Q.; Thang, M.W.C.; Le, L.; Nuhn, M.; Herzig, V.; Saez, N.J.; Cristofori-Armstrong, B.; et al. ArachnoServer 3.0: An online resource for automated discovery, analysis and annotation of spider toxins. Bioinformatics 2018, 34, 1074-1076. [CrossRef] [PubMed]

3. Escoubas, P. Molecular diversification in spider venoms: A web of combinatorial peptide libraries. Mol. Divers. 2006, 10, 545-554. [CrossRef] [PubMed]

4. Klint, J.K.; Senff, S.; Rupasinghe, D.B.; Er, S.Y.; Herzig, V.; Nicholson, G.M.; King, G.F. Spider-venom peptides that target voltage-gated sodium channels: Pharmacological tools and potential therapeutic leads. Toxicon 2012, 60, 478-491. [CrossRef] [PubMed]

5. King, G.F.; Hardy, M.C. Spider-venom peptides: Structure, pharmacology, and potential for control of insect pests. Annu. Rev. Entomol. 2013, 58, 475-496. [CrossRef] [PubMed]

6. Peigneur, S.; de Lima, M.E.; Tytgat, J. Phoneutria nigriventer venom: A pharmacological treasure. Toxicon 2018, 151, 96-110. [CrossRef] [PubMed]

7. Herzig, V.; John Ward, R.; Ferreira dos Santos, W. Intersexual variations in the venom of the Brazilian 'armed' spider Phoneutria nigriventer (Keyserling, 1891). Toxicon 2002, 40, 1399-1406. [CrossRef]

8. Bucaretchi, F.; Mello, S.M.; Vieira, R.J.; Mamoni, R.L.; Blotta, M.H.; Antunes, E.; Hyslop, S. Systemic envenomation caused by the wandering spider Phoneutria nigriventer, with quantification of circulating venom. Clin. Toxicol. 2008, 46, 885-889. [CrossRef] [PubMed] 
9. Diniz, M.R.V.; Paiva, A.L.B.; Guerra-Duarte, C.; Nishiyama, M.Y., Jr.; Mudadu, M.A.; Oliveira, U.; Borges, M.H.; Yates, J.R.; Junqueira-de-Azevedo, I.L. An overview of Phoneutria nigriventer spider venom using combined transcriptomic and proteomic approaches. PLoS ONE 2018, 13, e0200628. [CrossRef] [PubMed]

10. Diniz, C.R.; Cordeiro Mdo, N.; Junor, L.R.; Kelly, P.; Fischer, S.; Reimann, F.; Oliveira, E.B.; Richardson, M. The purification and amino acid sequence of the lethal neurotoxin Tx1 from the venom of the Brazilian 'armed' spider Phoneutria nigriventer. FEBS Lett. 1990, 263, 251-253. [CrossRef]

11. King, G.F.; Gentz, M.C.; Escoubas, P.; Nicholson, G.M. A rational nomenclature for naming peptide toxins from spiders and other venomous animals. Toxicon 2008, 52, 264-276. [CrossRef] [PubMed]

12. Figueiredo, S.G.; Garcia, M.E.; Valentim, A.C.; Cordeiro, M.N.; Diniz, C.R.; Richardson, M. Purification and amino acid sequence of the insecticidal neurotoxin Tx4(6-1) from the venom of the 'armed' spider Phoneutria nigriventer (Keys). Toxicon 1995, 33, 83-93. [CrossRef]

13. Cordeiro Mdo, N.; Diniz, C.R.; Valentim Ado, C.; von Eickstedt, V.R.; Gilroy, J.; Richardson, M. The purification and amino acid sequences of four Tx2 neurotoxins from the venom of the Brazilian 'armed' spider Phoneutria nigriventer (Keys). FEBS Lett. 1992, 310, 153-156. [CrossRef]

14. Matavel, A.; Fleury, C.; Oliveira, L.C.; Molina, F.; de Lima, M.E.; Cruz, J.S.; Cordeiro, M.N.; Richardson, M.; Ramos, C.H.; Beirao, P.S. Structure and activity analysis of two spider toxins that alter sodium channel inactivation kinetics. Biochemistry 2009, 48, 3078-3088. [CrossRef] [PubMed]

15. Matavel, A.; Cruz, J.S.; Penaforte, C.L.; Araujo, D.A.; Kalapothakis, E.; Prado, V.F.; Diniz, C.R.; Cordeiro, M.N.; Beirao, P.S. Electrophysiological characterization and molecular identification of the Phoneutria nigriventer peptide toxin PnTx2-6. FEBS Lett. 2002, 523, 219-223. [CrossRef]

16. Catterall, W.A.; Cestele, S.; Yarov-Yarovoy, V.; Yu, F.H.; Konoki, K.; Scheuer, T. Voltage-gated ion channels and gating modifier toxins. Toxicon 2007, 49, 124-141. [CrossRef] [PubMed]

17. Silva, C.N.; Nunes, K.P.; Torres, F.S.; Cassoli, J.S.; Santos, D.M.; Almeida Fde, M.; Matavel, A.; Cruz, J.S.; Santos-Miranda, A.; Nunes, A.D.; et al. PnPP-19, a Synthetic and Nontoxic Peptide Designed from a Phoneutria nigriventer Toxin, Potentiates Erectile Function via NO/cGMP. J. Urol. 2015, 194, 1481-1490. [CrossRef] [PubMed]

18. Nunes, K.P.; Cordeiro, M.N.; Richardson, M.; Borges, M.N.; Diniz, S.O.; Cardoso, V.N.; Tostes, R.; De Lima, M.E.; Webb, R.C.; Leite, R. Nitric oxide-induced vasorelaxation in response to PnTx2-6 toxin from Phoneutria nigriventer spider in rat cavernosal tissue. J. Sex. Med. 2010, 7, 3879-3888. [CrossRef] [PubMed]

19. Nunes, K.P.; Torres, F.S.; Borges, M.H.; Matavel, A.; Pimenta, A.M.; De Lima, M.E. New insights on arthropod toxins that potentiate erectile function. Toxicon 2013, 69, 152-159. [CrossRef] [PubMed]

20. Alewood, D.; Birinyi-Strachan, L.C.; Pallaghy, P.K.; Norton, R.S.; Nicholson, G.M.; Alewood, P.F. Synthesis and characterization of delta-atracotoxin-Ar1a, the lethal neurotoxin from venom of the Sydney funnel-web spider (Atrax robustus). Biochemistry 2003, 42, 12933-12940. [CrossRef] [PubMed]

21. Deuis, J.R.; Mueller, A.; Israel, M.R.; Vetter, I. The pharmacology of voltage-gated sodium channel activators. Neuropharmacology 2017, 127, 87-108. [CrossRef] [PubMed]

22. Wingerd, J.S.; Mozar, C.A.; Ussing, C.A.; Murali, S.S.; Chin, Y.K.; Cristofori-Armstrong, B.; Durek, T.; Gilchrist, J.; Vaughan, C.W.; Bosmans, F.; et al. The tarantula toxin beta/delta-TRTX-Pre1a highlights the importance of the12 S-S voltage-sensor region for sodium channel subtype selectivity. Sci. Rep. 2017, 7, 974. [CrossRef] [PubMed]

23. Leipold, E.; Hansel, A.; Borges, A.; Heinemann, S.H. Subtype specificity of scorpion beta-toxin Tz1 interaction with voltage-gated sodium channels is determined by the pore loop of domain 3. Mol. Pharmacol. 2006, 70, 340-347. [CrossRef] [PubMed]

24. Nicholson, G.M.; Little, M.J.; Birinyi-Strachan, L.C. Structure and function of delta-atracotoxins: Lethal neurotoxins targeting the voltage-gated sodium channel. Toxicon 2004, 43, 587-599. [CrossRef] [PubMed]

25. Peigneur, S.; Cologna, C.T.; Cremonez, C.M.; Mille, B.G.; Pucca, M.B.; Cuypers, E.; Arantes, E.C.; Tytgat, J. A gamut of undiscovered electrophysiological effects produced by Tityus serrulatus toxin 1 on NaV-type isoforms. Neuropharmacology 2015, 95, 269-277. [CrossRef] [PubMed]

26. Leipold, E.; Borges, A.; Heinemann, S.H. Scorpion beta-toxin interference with NaV channel voltage sensor gives rise to excitatory and depressant modes. J. Gen. Physiol. 2012, 139, 305-319. [CrossRef] [PubMed] 
27. Rash, L.D.; Birinyi-Strachan, L.C.; Nicholson, G.M.; Hodgson, W.C. Neurotoxic activity of venom from the Australian eastern mouse spider (Missulena bradleyi) involves modulation of sodium channel gating. Br. J. Pharmacol. 2000, 130, 1817-1824. [CrossRef] [PubMed]

28. Gunning, S.J.; Chong, Y.; Khalife, A.A.; Hains, P.G.; Broady, K.W.; Nicholson, G.M. Isolation of delta-missulenatoxin-Mb1a, the major vertebrate-active spider delta-toxin from the venom of Missulena bradleyi (Actinopodidae). FEBS Lett. 2003, 554, 211-218. [CrossRef]

29. Nicholson, G.M.; Willow, M.; Howden, M.E.; Narahashi, T. Modification of sodium channel gating and kinetics by versutoxin from the Australian funnel-web spider Hadronyche versuta. Pflugers Arch. 1994, 428, 400-409. [CrossRef] [PubMed]

30. Nicholson, G.M.; Little, M.J.; Tyler, M.; Narahashi, T. Selective alteration of sodium channel gating by Australian funnel-web spider toxins. Toxicon 1996, 34, 1443-1453. [CrossRef]

31. Gilles, N.; Harrison, G.; Karbat, I.; Gurevitz, M.; Nicholson, G.M.; Gordon, D. Variations in receptor site-3 on rat brain and insect sodium channels highlighted by binding of a funnel-web spider delta-atracotoxin. Eur. J. Biochem. 2002, 269, 1500-1510. [CrossRef] [PubMed]

32. Paiva, A.L.; Matavel, A.; Peigneur, S.; Cordeiro, M.N.; Tytgat, J.; Diniz, M.R.; de Lima, M.E. Differential effects of the recombinant toxin PnTx4(5-5) from the spider Phoneutria nigriventer on mammalian and insect sodium channels. Biochimie 2016, 121, 326-335. [CrossRef] [PubMed]

33. Peigneur, S.; Billen, B.; Derua, R.; Waelkens, E.; Debaveye, S.; Beress, L.; Tytgat, J. A bifunctional sea anemone peptide with Kunitz type protease and potassium channel inhibiting properties. Biochem. Pharmacol. 2011, 82, 81-90. [CrossRef] [PubMed]

(c) 2018 by the authors. Licensee MDPI, Basel, Switzerland. This article is an open access article distributed under the terms and conditions of the Creative Commons Attribution (CC BY) license (http://creativecommons.org/licenses/by/4.0/). 